\title{
Structure and Properties of the Triplet State of Styrene Derivatives Studied by Laser-Flash-Photolysis
}

\section{R. BONNEAU and B. HERRAN}

\author{
E.R.A. No. 167 DU CNRS, Laboratoire de Chimie Physique A, Université de \\ Bordeaux I, 33405 Talence Cedex, France
}

(Received 18 March 1983; in final form 20 May 1983)

The triplet state of a series of compounds, each containing the styrene moiety, has been studied by nanosecond laser flash photolysis. The lifetimes, the TT absorption spectra and the rate constants for deactivation by several quenchers have been determined. It is shown from these results that, in the triplet state of styrene derivatives in their lowest energy conformation, the double bond is twisted by $90^{\circ}$ for flexible molecules (perpendicular triplet) or by an angle as large as allowed by the molecular framework of rigid molecules (twisted triplet). There is no equilibrium between planar and perpendicular (or twisted) triplets, in contrast with the case of stilbene. The influence of substituants on the lifetime of the perpendicular triplet and the mechanism of quenching of this species by oxygen have been investigated.

The triplet states of several ethylenic compounds have been observed during the last few years by laser-flash-photolysis. Transient absorptions assigned to a distorted conformation of the triplet state in which the double bond is twisted by $90^{\circ}$, the so-called "perpendicular" or "phantom" triplet, have been reported for $\alpha, \beta$-enones, ${ }^{1,2}$ stilbene $^{3}$ and styrene derivatives. ${ }^{4}$ These triplet species have very short lifetimes ranging from $10-100$ ns but, with very few exceptions, ${ }^{2,5}$ little is known about the relation between the lifetime and the molecular structure. Oxygen quenches the perpendicular triplet states very efficiently, not by the well-known energy transfer mechanism, but through a "spin exchange" mechanism ${ }^{6}$ which still needs more detailed investigation. Ethylenic perpendicular triplets may be considered as 1,2-triplet biradicals and it should be possible to support 
this biradical character by the effect of substituents or of specific reactants.

Here we report some properties of the triplet state of a series of styrene derivatives as measured by laser-flash-photolysis. These results give some insight into the relation between the lifetime and the molecular structure, the mechanism of quenching by oxygen and the biradical character of the perpendicular triplet.

\section{EXPERIMENTAL}

These compounds not commercially available were prepared by Grignard addition to (or reduction of) the parent ketone followed by dehydration of the alcohol. Since the intersystem-crossing quantum yield was assumed to be very low for styrene derivatives, ${ }^{7}$ the triplet state was populated by sensitization with xanthone or thioxanthone. The concentration of the styrene acceptor was $5 \times 10^{-2}$ to $10^{-1} \mathrm{M}$, giving a very fast energy transfer. Thus the main part of the decay of the acceptor triplet state was not perturbed by the energy transfer process and could be used for determining the triplet lifetime. Computer simulations indicate that with a concentration of acceptor equal to $10^{-1} \mathrm{M}$ and a rate constant for energy transfer equal to $5 \times$ $10^{9} \mathrm{M}^{-1} \mathrm{~s}^{-1}$ the main part of the decay of the observed transient absorption could be used for determination of the lifetime as long as the triplet lifetime was larger than $10 \mathrm{~ns}$. The laser-flash-photolysis apparatus, built around a "Quantel" $\mathrm{Nd}^{+++}$laser, has already been described. ${ }^{2}$ The excitation wavelength was $353 \mathrm{~nm}$.

\section{RESULTS}

The compounds studied can be classified into three groups:

(i) "Freely rotating styrenes", in which the double bond can twist by $180^{\circ}$ : styrene, $\alpha$-methylstyrene ( $\alpha$-MS); trans- $\beta$-methylstyrene $(\beta$-MS); trans- $\beta$-cyanostyrene ( $\beta$-CS); 4-methylstyrene (4-MS); 2,6-dichlorostyrene (DCS) and stilbene.

(ii) "Flexible styrenes" in which the double bond can twist by more than $90^{\circ}$ to give unstable "trans" isomer:" 1 -phenylcyclohexene (PC6) and 1-phenylcycloheptene (PC7). 
(ii) "Rigid styrenes" in which the double bond cannot twist by a large angle: 2-phenyl 2-norborene (PN), and 1,2-dihydronaphthalene (DHN).

It will be shown that benzosuberene (BS) must be classified in the second group.

\section{(a) Absorption spectra}

Upon sensitization by thioxanthone in benzene, toluene or cyclohexane as solvent, each compound gave rise to a transient absorption in the $310-350 \mathrm{~nm}$ region. Measurements were possible only within these two limits because of the strong absorption of the solution in the $350-380 \mathrm{~nm}$ range and below $310 \mathrm{~nm}$. For the rigid styrenes, PN and DHN, the transient absorption extended to wavelength longer than $360 \mathrm{~nm}$ and the absorption spectra for $\lambda>360 \mathrm{~nm}$ were obtained by using xanthone as sensitizer. Figure 1 shows typical absorption

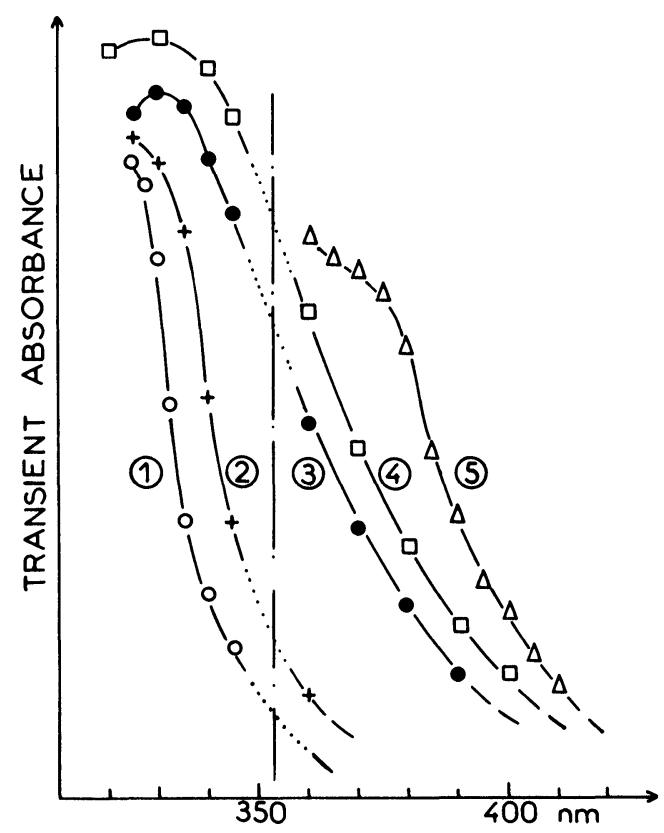

FIGURE 1 Absorption spectra of the triplet state of styrene (1), trans $\beta$-methylstyrene (2), dihydronaphthalene (3), 2-phenyl, 2-norbornene (4) and stilbene (5). 
spectra of the transient species observed for two free-rotating styrenes and two rigid styrenes. For PC6 and PC7 the transient absorption spectrum was similar to that of $\beta$-MS, slightly red shifted, but its determination was complicated by the absorption of the "trans" ground state.

For stilbene the transient absorption could be monitored only at wavelengths longer than $360 \mathrm{~nm}$, using xanthone as sensitizer. The observed transient absorption spectrum is very similar to that reported by Görner and Schulte-Frohlinde: ${ }^{3}$ it appears as the tail of a broad band, the maximum of which would be at $\lambda>360 \mathrm{~nm}$, without any structure contrary to what is observed in frozen solutions.

The extinction coefficients $(\varepsilon)$ were estimated by comparing the intensity of the transient absorptions at $325 \mathrm{~nm}$ with the absorption at $413 \mathrm{~nm}$ of triplet naphthalene produced under the same conditions of sensitization by thioxanthone. Since the concentrations of naphthalene and styrenes were large enough to ensure that energy transfer proceed in all cases with a yield very close to unity, the transient absorptions are proportional to the $\varepsilon$ values and, with $\varepsilon_{413}=24000$ for naphthalene triplet in cyclohexane,${ }^{10}$ one gets the following values of $\varepsilon_{325}$ for the styrenic transient species: 2200 for styrene, 3500 for $\beta$-MS, 4400 for PC6 and 17900 for PN.

\section{(b) Lifetimes}

In argon flushed toluene solutions, the transient absorptions decayed according to first order kinetics, with lifetimes as listed in Table I.

Lifetime values longer than one microsecond are considered as minimum values since the measured lifetime may be limited by quenching by a small amount of impurities with low lying triplets which may have been present in the styrenes or by oxygen remaining after flushing the solution with argon for several minutes. However,

TABLE I

\begin{tabular}{cccccc}
\hline Compound & $(\mathrm{ns})$ & Compound & $(\mathrm{ns})$ & Compound & (ns) \\
\hline Styrene & 24 & $\beta$-CS & 66 & BS & 60 \\
$\beta$-MS & 27 & DCS & $<7$ & DHN & $>1500$ \\
$\alpha$-MS & 30 & PC7 & 28 & PN & $>2500$ \\
4 -MS & 31 & PC6 & 65 & $t$-stilbene & 52 \\
\hline
\end{tabular}


the concentrations of oxygen and/or impurities were certainly too low for significant quenching of species with lifetimes shorter than $100 \mathrm{~ns}$.

\section{(c) Addition of quenchers}

Oxygen increases the rate of decay of all the transient absorptions reported above. Quenching rate constants were calculated from the lifetimes measured in argon-flushed, aerated and oxygen-saturated solutions. For some compounds, we have also measured the quenching rate constants by ferrocene, 1.3.5-hexatriene, 1.3-cyclohexadiene and biacetyl, a series of quenchers with a triplet energy lower than the energy of the Franck-Condon triplet of styrene $(62 \mathrm{kcal} / \mathrm{mol})$ and with very low extinction coefficients at 325 and $353 \mathrm{~nm}$. These quenching rate constants, measured in toluene solutions, are listed in Table II.

The quenching rate constants by nitric oxide of the PC6, PC7 and PN transients and of the anthracene triplet were measured by adding known pressures of NO in a cell containing a degassed cyclohexane solution, then shaking and sealing the cell and measuring the transient lifetime. The solubility of NO in cyclohexane was assumed to be $1.3 \times 10^{-2} \mathrm{M}$ under one atmosphere pressure. ${ }^{11}$

For PC6 and PC7, the intensity of the absorption of the "trans" ground state, $\mathrm{OD}_{\mathrm{tr}}$, was measured at $300 \mathrm{~ns}$ after the excitation as a function of the $\mathrm{O}_{2}$ concentration. Relative yields of formation of the "trans" ground state were then obtained from the measured $\mathrm{OD}_{\mathrm{tr}}$, after normalization of a constant energy of excitation and correction for the partial quenching of the triplet sensitizer by $\mathrm{O}_{2}$. As the $\mathrm{O}_{2}$ concentration increased, both the yield of formation of the "trans" ground state, $\phi$, and the lifetime of the transient, $\tau$, decreased but here was no direct proportionality between $\phi$ and $\tau$.

For PC6 and $\beta$-CS, the effect of $\mathrm{O}_{2}$ on the $\phi$ value was measured by excitation under continuous irradiation in the presence of a sensitizer and g.c. analysis of the products. Since the "trans" PC6 has a short lifetime $(\sim 10 \mu \mathrm{s})$, and cannot be directly detected by g.c., it was converted into the corresponding methyl ether by using acidic methanol as solvent, ${ }^{12}$ triphenylene as sensitizer and irradiation at $334 \mathrm{~nm}$; the yield of conversion from "trans" PC6 to the ether was then calculated from the known rate constants. ${ }^{8}$ The values of $\tau / \tau_{0}$ 
R. BONNEAU AND B. HERRAN

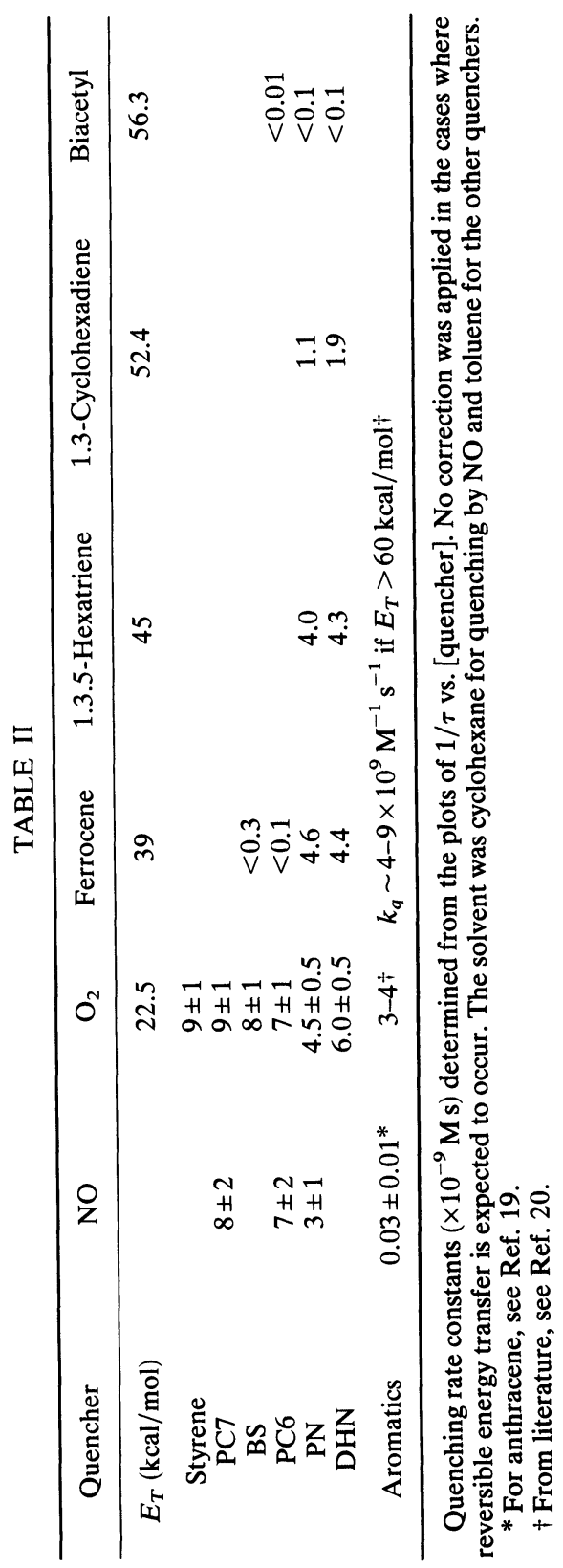


and those of $\phi / \phi_{0}$ obtained by the two methods are listed in Table III. The two sets of $\phi / \phi_{0}$ values obtained for PC6 are in good agreement.

\section{DISCUSSION}

\section{(a) Nature and geometry of the observed transient}

In all cases the observed transient absorption is assigned to the triplet state produced by sensitization but, as shown by the above results, the properties of this triplet state depend greatly on the flexibility of the molecular structure. When the double bond is embedded in a rigid structure, so that the molecular conformation of the triplet is necessarily close to that of the ground state, the lifetime of the transient is longer than a microsecond. In contrast, when the double bond can twist by $90^{\circ}$ or more, the lifetime of the transient is shorter than $70 \mathrm{~ns}$. However in both cases the transient absorption appears in the same wavelength range and the absorption spectra are similar. This similarity can be rationalized by one of the following possibilities:

(i) The transient absorption is related in all cases to the planar triplet ${ }^{3} t$ or ${ }^{3} c$, the very short lifetime of this species in flexible systems being due to irreversible twisting of the double bond to give the perpendicular triplet ${ }^{3} p$.

(ii) The transient absorption is related only to the planar triplet which would be, for flexible systems, in fast equilibrium with a very short lived perpendicular triplet.

(iii) The observed absorption is related to a planar (or nearly planar) triplet state for rigid compounds but to a perpendicular triplet in the case of flexible molecules.

The first hypothesis can easily be ruled out. In the presence of oxygen the lifetime of the planar triplet, $\tau$, and the yield of isomerisation, $\phi$, would be:

$$
\tau=1 /\left(k_{1}+k_{t w}+k_{q}\left|\mathrm{O}_{2}\right|\right)
$$

and

$$
\phi=\varphi k_{t w} /\left(k_{1}+k_{t w}+k_{q}\left|\mathrm{O}_{2}\right|\right)=\varphi \cdot k_{t w} \cdot \tau .
$$

with $k_{q}, k_{t w}$ and $k_{1}$ being respectively the rate constants for quenching by $\mathrm{O}_{2}$, twisting to ${ }^{2} p$ and decay to the ground state from the planar 


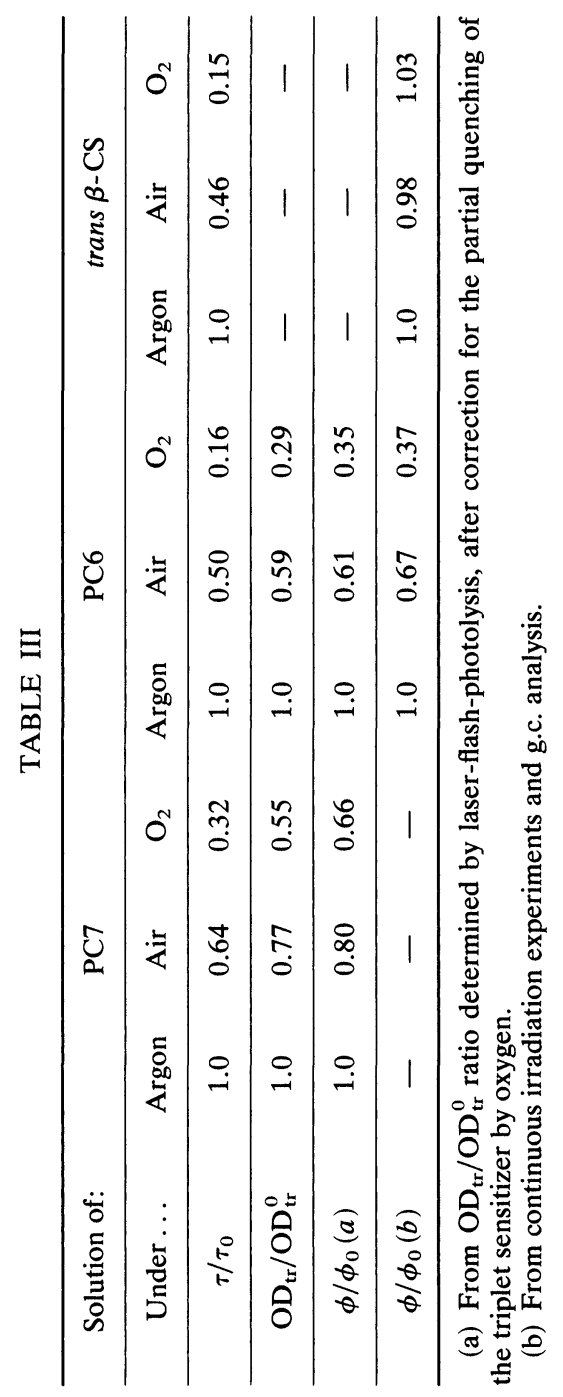


triplet and $\varphi$ the yield of isomerization from ${ }^{3} p$. Addition of oxygen would decrease both $\phi$ and $\tau$ and there would be a direct proportionnality between $\phi$ and $\tau$ : this is not the case as shown from Table III. Furthermore, the transient of rigid and flexible styrenes should be quenched by low energy acceptors such as ferrocene or 1.3.5hexatriene with the same rate constant, $k_{q} \simeq 4-5 \times 10^{9} \mathrm{M}^{-1} \mathrm{~s}^{-1}$ : Table II shows that this is not the case. The value of the quenching rate constant by $\mathrm{O}_{2}$ and the effect of chlorine substituent on the lifetime are additional reasons for discarding this hypothesis.

It is not so easy to choose between the second and third possible interpretations, especially if the equilibrium involved in the second hypothesis is largely in favour of ${ }^{3} p$. The behavior of the transient will be determined either mainly (in the case of (ii)) or exclusively (in the case of (iii)) by the properties of ${ }^{3} p$.

Since the existence of an equilibrium between the planar and perpendicular conformations of the stilbene triplet state has been demonstrated, ${ }^{3}$ the possibility of such an equilibrium for styrenes-or at least for some styrene derivatives-has to be carefully considered. Let us consider the following points:

(1) the fact that the $T-T$ absorptions of rigid and flexible styrenes appear in the same wavelength range but with a lower extinction coefficient for the flexible systems could be explained by assuming that only the fraction of the triplet under planar conformation gives rise to the observed absorption. Then the percentage of the planar triplet would be directly related to the apparent extinction coefficient, decreasing from $100 \%$ for the rigid $\mathrm{PN}\left(\varepsilon_{325} \simeq 18000\right)$ to $24 \%$ for the flexible but strained PC6 $\left(\varepsilon_{325}=4400\right)$ and to $12 \%$ for the freely rotating styrene $\left(\varepsilon_{325} \simeq 2200\right)$. But alternatively, assuming that the transient absorption observed for flexible molecules originates from ${ }^{3} p$, the increase of the extinction coefficient at $325 \mathrm{~nm}$ from 2200 for styrene to 3500 for $\beta$-MS and 4400 for PC6 can easily be explained by hyperchromic and bathochromic effects of the alkylsubstitution. The difference between $\varepsilon_{325}$ for PC6 and PN may be explained by the different nature of the TT transitions, occurring from ${ }^{3} p$ in PC6 and from planar triplet in PN, the similarity of the absorption spectra being coincidental;

(2) the significance of the lack of an effect of azulene on the photostationary ratio cis/trans $\beta$-methylstyrene, presented by Caldwell $^{5,13}$ as a proof of the very low population of the planar triplet, 
may not be significant since, if an equilibrium ${ }^{3} t \rightleftarrows^{3} p$ exists, another equilibrium ${ }^{3} p \rightleftarrows{ }^{3} c$ should be considered. Indeed with a double equilbrium, ${ }^{3} t \rightleftarrows{ }^{3} p \rightleftarrows{ }^{3} c$, and assuming that ${ }^{3} t$ and ${ }^{3} c$ are exactly isoenergetic, there should be no effect of azulene on the photostationary ratio. But as long as the energy difference between ${ }^{3} c$ and ${ }^{3} t$ is larger than $1 \mathrm{kcal} / \mathrm{mole}$, the "azulene effect" should be quite sensitive to detect the existence of a planar triplet in a non-negligible amount (see Appendix A). Since the steric hindrance in cis $\beta$-MS most probably raises the energy of ${ }^{3} c$ by more than $1 \mathrm{kcal} / \mathrm{mol}$ above that of ${ }^{3} t$, the conclusions of Caldwell, ${ }^{5}$ according to which the percentage of planar triplet must be less than $5 \%$ in $\beta$-MS, are validated. Therefore the transient absorption for this compound, which is only 5 times less intense than that observed for PN, cannot originate from a planar triplet: it is a specific absorption of the perpendicular triplet;

(3) in phenylcycloalkenes such as PC6 and PC7, twisting of the double bond by a $90^{\circ}$ angle involves an appreciable strain energy which raises the energy of ${ }^{3} \mathrm{p}$, greatly increasing the probability of an equilibrium. This effect is expected to give a percentage of planar triplet, ${ }^{3} c$, much larger for PC6 than for PC7. Qualitatively, this seems to explain why the yield of cis $\rightarrow$ "trans" isomerisation of these compounds decreases in presence of oxygen and why the decrease is larger for PC6 than for PC7 since the quenching of ${ }^{3} c$ by oxygen gives exclusively the cis ground state. But a quantitative analysis given in Appendix B shows that the decrease of $\phi_{c t}$ in presence of oxygen cannot be explained by the existence of an equilibrium between ${ }^{3} c$ and ${ }^{3} p$;

(4) the lifetime of the triplet of PC6 is about twice that measured for PC7. Similar results have been obtained for 1-anisylcyclohexene and 1-anisyl-cycloheptene and this has been explained by a limitation of the twist angle of the cyclohexene ring to a value of $50-55^{\circ} .{ }^{5}$ This cannot be the right explanation since the "trans" PC6 is populated from the PC6 triplet with a yield of $0.34 \pm 0.07^{18}$ indicating clearly that the PC6 can be twisted up to $90^{\circ}$. Alternatively this could be explained by assuming a rapid equilibrium between ${ }^{3} c$ (with a lifetime longer than $1 \mu \mathrm{s}$ ) and ${ }^{3} p$ (with a 30 ns lifetime) in $1: 1$ ratio in the case of cyclohexene derivatives whereas the triplet would be entirely in the perpendicular conformation for cycloheptene derivatives. Then the triplet state of PC6 should be quenched by ferrocene with a rate constant which would be about $2 \times 10^{9} \mathrm{M}^{-1} \mathrm{~s}^{-1}$, i.e., one half of the 
rate constant of quenching of "rigid styrenes" triplet by ferrocene. This is not the case since the ferrocene does not quench the triplet of PC6-or quenches it with a rate constant lower than $10^{8} \mathrm{M}^{-1} \mathrm{~s}^{-1}$. Therefore the percentage of planar triplet is less than $2 \%$ in the case of PC6, the compound in which the large strain energy induced by twisting of the cyclohexene ring is the most favorable to the existence of a planar triplet. Thus one can ascertain that, for PC7 and "freerotating", the triplet state is entirely in the perpendicular conformation.

The very short lifetime of the BS triplet state and the inefficient quenching of this species by ferrocene indicate that BS is a highly flexible molecule in which the triplet can reach a perpendicular conformation. We believe that BS is able to give an unstable "trans" ground state (A in Figure 2) which, however, could not be detected by absorption. But, during a study of the triplet state of a series of $\alpha$-naphthyl-ethylenic compounds, ${ }^{14}$ we could observe an absorption characteristic of the unstable "trans" 3-( $\alpha$-naphthyl)1.2-benzosuberene ( $\mathbf{B}$ in Figure 2). Examining the structures of $\mathbf{A}$ and $\mathbf{B}$, it is easly understood why $\mathbf{A}$ does not appear in absorption whereas $\mathbf{B}$ does: in A the $\pi$-systems of the "trans" double bond and of the benzo ring are very weakly coupled; in B that is also true but the "trans" double bond is strongly coupled with the $\alpha$-naphthyl $\pi$-system.

Considering now the "rigid" PN and DHN, it appears that the triplet state of these molecules can suffer an appreciable relaxation with respect to the ground state geometry. The energy of the FranckCondon triplet of these molecules is expected to be slightly lower than that of styrene, i.e., about $60-62 \mathrm{kcal} / \mathrm{mol}$. But the values of the quenching rate constants reported in Table II suggest that the energy of the relaxed triplet is about $50-42 \mathrm{kcal} / \mathrm{mol}$. Most probably,

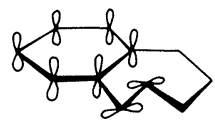

(A)

(B)

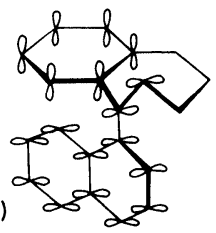

FIGURE 2 Postulated geometries of the unstable "trans", ground state of benzosuberene (A) and $\alpha$-naphthyl-bensozuberene (B) with a schematic representation of the $\pi$-orbitals. 
the relaxation process which accounts for this $8-12 \mathrm{kcal} / \mathrm{mol}$ energy loss is the twisting of the double bond. For norbornene, a similar energy loss of $15 \mathrm{kcal} / \mathrm{mol}$ has been reported ${ }^{15}$ and explained by twisting of the double bond. If that interpretation is correct, the energy loss comes mainly from an increase of the energy of the ground state and, for a smaller part, from a decrease of the triplet energy but, anyway, this should correspond to an appreciable twist angle.

Therefore it seems that, in styrenic compounds, the ground geometry corresponds to an energy maximum for the triplet state which, in all cases, relaxes as much as possible by twisting of the double bond as shown in Figure 3.

\section{(b) Lifetime and substituents}

The triplet lifetimes measured for styrene and methyl-styrenes are in very good agreement with those measured by a quite different method for 4-methoxy-styrene and its methylated derivatives. ${ }^{5,16}$ Both series of measurements indicate that methylation in the $\alpha$ or $\beta$ position increases the lifetime from $22-24 \mathrm{~ns}$ to $28-31 \mathrm{~ns}$. This increase has been attributed to a decrease in the number of the vinyl $\mathrm{CH}$ bonds (the motion of which would induce the intersystem-crossing ${ }^{3} p \rightarrow p_{0}$ ) rather than to hyperconjugative delocalisation. ${ }^{5}$ Yet, increasing the average distance between the unpaired electrons, which hyperconju-

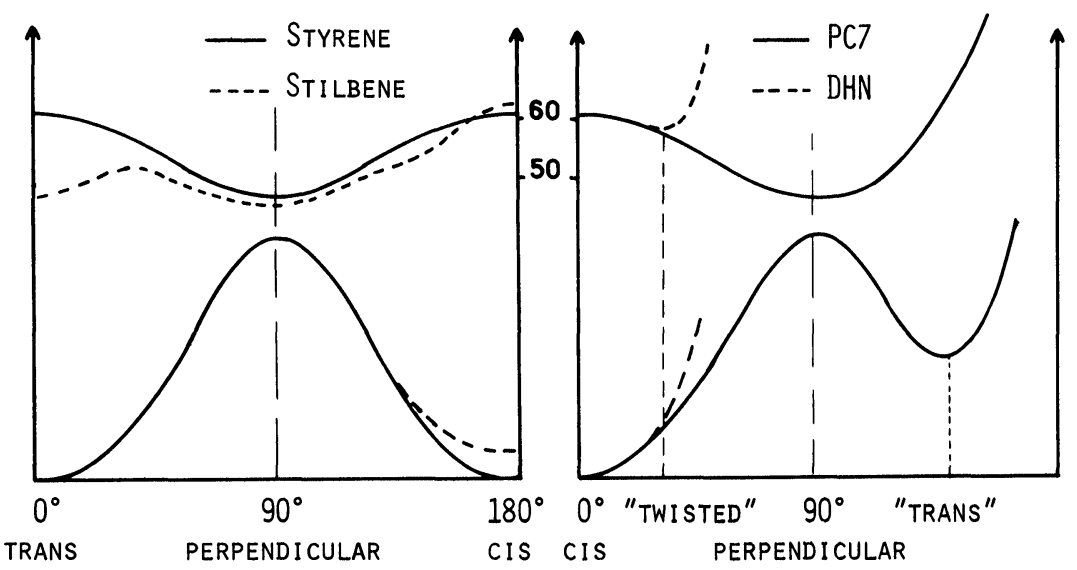

FIGURE 3 Schematic potential energy surfaces for the ground state and the lowest state of several styrene derivatives as a function of the twist angle of the double bond. 
gation should do, seems to be an efficient way for increasing the triplet lifetime: the difference between the triplet lifetimes of $\beta$-CS $(66 \mathrm{~ns})$ and $\beta$-MS $(27 \mathrm{~ns})$ is most probably due to the strong attractive effect of the CN substituent on the electron initially located on the $\beta$ carbon. For 1,4-triplet biradicals derived from valerophenone the lifetime is around $100 \mathrm{~ns}^{17}$

The lifetime of the perpendicular triplet is also increased by stabilizing the two radical moieties: for stilbene, in which each radical part is stabilized by a phenyl, the triplet lifetime is about twice as large as for styrene.

The presence of chlorine atoms in the 2 and 6 positions of styrene reduces the triplet lifetime from $24 \mathrm{~ns}$ to less than $7 \mathrm{~ns}$. This is a considerable effect for one of the lightest among the "heavy atoms", especially considering that the difference between the reciprocal lifetimes is more than $10^{8} \mathrm{~s}^{-1}$. This is related to the fact that the perpendicular triplet and ground states are very close in energy so that the process ${ }^{3} p \rightarrow p_{0}$ is quite comparable to intersystem crossing between the excited singlet and triplet manifolds of most aromatic molecules: here too, chlorination usually increases $k_{\text {isc }}$ by $10^{8}-10^{9} \mathrm{~s}^{-1}$.

\section{(c) Quenching by $\mathrm{NO}$ and $\mathrm{O}_{2}$}

The perpendicular triplet of flexible styrenes is quenched by NO with a large rate constant $\left(8 \pm 2 \times 10^{9} \mathrm{M}^{-1} \mathrm{~s}^{-1}\right)$ whereas for the triplet state of anthracene, considered as a model of aromatics, the rate constant is only $3 \pm 1 \times 10^{7} \mathrm{M}^{-1} \mathrm{~s}^{-1} \cdot{ }^{19}$ This may be explained by considering the strong biradical character of ${ }^{3} p$ and assuming that one (or both) radical part(s) can react with the NO radical at a nearly diffusion controlled rate. The triplet state of $\mathrm{PN}$ is quenched by NO with rather large rate constant, around $3 \times 10^{9} \mathrm{M}^{-1} \mathrm{~s}^{-1}$. This may be explained by considering that, as stated above, the norbornene double bond can be twisted in the triplet state by an angle much smaller than $90^{\circ}$ but large enough for giving to the triplet some biradical character.

For flexible styrenes, the energy gap between ${ }^{3} p$ and $p_{0}$ is very small and the quenching by oxygen cannot occur via the energy transfer mechanism (path III is scheme A). The efficient quenching of ${ }^{3} p$ by oxygen must occur via the "spin-exchange mechanism" (path I in scheme A) with a rate constant equal to $k_{\text {diff }} / 3$. $^{6}$ Measured rate constants, $k_{2}=9 \pm 1 \times 10^{9} \mathrm{M}^{-1} \mathrm{~s}^{-1}$ are indeed about one third of the 


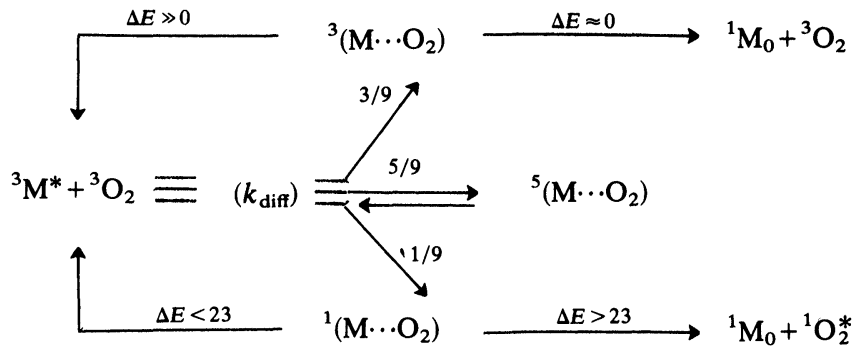

(With $\Delta E=$ energy difference between ${ }^{3} M^{*}$ and ${ }^{1} M_{0}$, in $\mathrm{kcal} / \mathrm{mol}$ )

(Scheme A)

diffusion rate constant since $k_{\text {diff }} \simeq 3 \times 10^{10} \mathrm{M}^{-1} \mathrm{~s}^{-1}$ in benzene and toluene. $^{20}$

The lifetime of the complex ${ }^{3}\left(\mathrm{M} \cdots \mathrm{O}_{2}\right)^{*}$ appearing in scheme $\mathrm{A}$ is probably extremely short and, up to now, no experimental evidence has been obtained to confirm its existence. We believe that the fact that oxygen does not change the yield of production of the cis and trans isomers from ${ }^{3} p$ in the case of $\beta$-CS whereas it does in the case of PC6 and PC7 could be explained by assuming that the ${ }^{3}\left(\mathrm{M} \cdots \mathrm{O}_{2}\right)^{*}$ complex is not only a transition state but has some energy of stabilisation. The decrease of $\phi_{c t}$, the yield of formation of the "trans" PC6 or PC7 from the triplet, in presence of oxygen can be explained by considering the following mechanistic scheme:

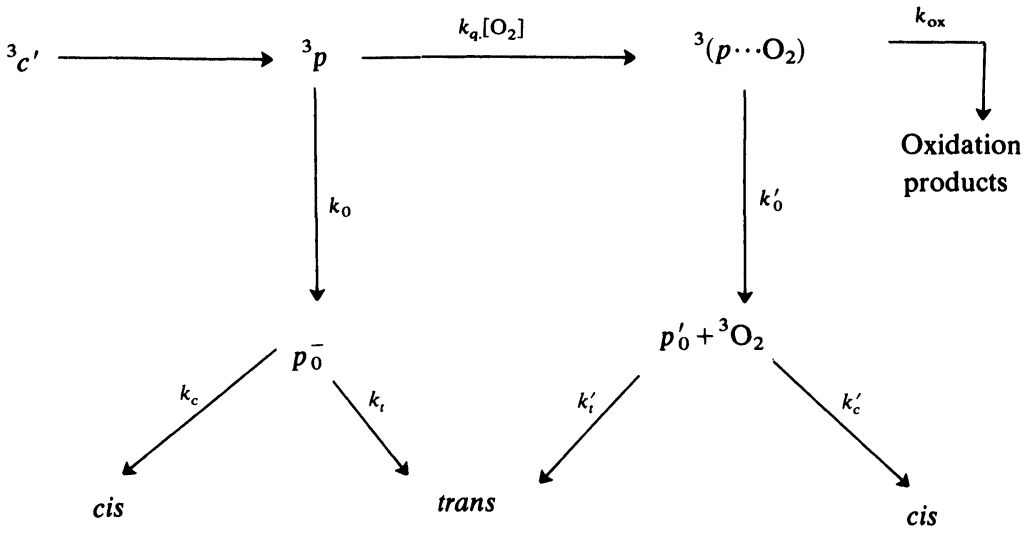

Note: For $\beta$-CS, cis and trans species have to be exchanged in this scheme.

(Scheme B) 
The triplet lifetime, $\tau$, and the yield of isomerization, $\phi$, in the absence and in presence of oxygen are respectively given by:

$$
\begin{aligned}
& \tau_{0}=1 / k_{0} \quad \text { and } \phi_{0}=k_{t} /\left(k_{t}+k_{c}\right) \\
& \tau=1 /\left(k_{0}+k_{q}\left[\mathrm{O}_{2}\right]\right) \text { and } \phi=\tau\left(k_{0} \phi_{0}+k_{q}\left[\mathrm{O}_{2}\right] \phi_{1}\right)
\end{aligned}
$$

with $\phi_{1}=\left[k_{0}^{\prime} /\left(k_{0}^{\prime}+k_{\mathrm{ox}}\right)\right]\left[k_{t}^{\prime} /\left(k_{t}^{\prime}+k_{c}^{\prime}\right)\right]$ being the yield of isomerization from the $\left({ }^{3} p \cdots \mathrm{O}_{2}\right)$ complex. Then one gets:

$$
\phi / \phi_{0}=\phi_{1} / \phi_{0}+\left(1-\phi_{1} / \phi_{0}\right) \times \tau / \tau_{0}
$$

Experimental values of $\phi / \phi_{0}$ and $\tau / \tau_{0}$ given in Table III do in fact obey this relation, as shown on Figure 4, giving the following values for the $\phi_{1} / \phi_{0}$ ratio: 1.0 for $\beta$-CS, 0.48 for PC7 and 0.24 for PC6. Thus for cyanostyrene $\phi_{1}=\phi_{0}$ and, according to this scheme, this means that formation of oxidation products is negligible $\left(k_{\mathrm{ox}} \ll k_{0}^{\prime}\right)$ and $p_{0}^{\prime}$ yields the cis and trans isomers in the same ratio as $p_{0}$. Assuming that, for PC6 and PC7 as for $\beta$-CS, $k_{\mathrm{ox}} \ll k_{0}^{\prime}$ (and indeed no oxidation products were detected by g.c. after irradiation of $\mathrm{O}_{2^{-}}$ saturated solutions) it appears that the efficiency of producing the "trans" isomer is lower for $p_{0}^{\prime}$ than for $p_{0}$, by a factor of 2 for PC7 and a factor of 4 in the case of PC6. This seems to indicate that the

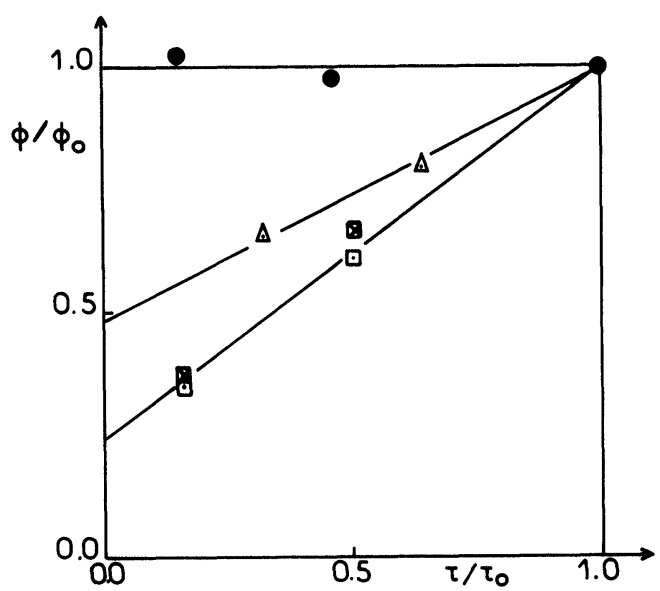

FIGURE 4 Relation between $\phi$, the quantum yield of sensitized cis-trans (or transcis) photoisomerization, and the triplet lifetime, $\tau$, measured in solutions containing various amounts of oxygen. - : trans $\beta$-cyanostyrene; $\triangle \longrightarrow$ : 1-phenykycloheptene; $\square \square$ : 1-phenylcyclohexene. See Table III and Section C of the Discussion. 
geometry of $p_{0}^{\prime}$ is different from that of $p_{0}$, probably due to some geometrical relaxation of ${ }^{3} p$ in the $\left({ }^{3} p \cdots \mathrm{O}_{2}\right)^{*}$ complex.

We think that the magnitude of the $\mathrm{O}_{2}$ effect must be related to the assymetry of the energy curve of the triplet with respect to a $90^{\circ}$ twist angle: for PC6 and PC7 such an assymetry is induced by the strain energy of the ring and this assymetry is of course greater for PC6 than for PC7. In contrast, if the energy curve is symmetric, interaction with oxygen will be symmetric too and $\phi$ is not changed: this is the case for $\beta$-CS and $\beta$-tertiobutyl styrene. ${ }^{21}$

\section{CONCLUSION}

For styrene derivatives, the ground state geometry corresponds to an energy maximum in the lowest triplet surface and the triplet state relaxes by twisting of the double bond as much as allowed by the molecular frame. This yields a twisted triplet for "rigid" molecules and a perpendicular triplet for "flexible" compounds. The observed $T-T$ absorption originates from these relaxed triplet conformations and there is no equilibrium between the planar and relaxed triplet states, in contrast with the case of stilbene.

The perpendicular triplet state has a strong biradical character and its lifetime, i.e., the rate of intersystem crossing between the triplet and ground state biradicals is extremely sensitive to spin-orbit coupling perturbations induced by heavy atoms. Oxygen quenches the perpendicular triplet via a ${ }^{3}\left(p \cdots \mathrm{O}_{2}\right)$ complex in which the interaction between ${ }^{3} p$ and $\mathrm{O}_{2}$ induces a geometrical relaxation of ${ }^{3} p$ which leads to a modification of the cis-trans isomerization quantum yield in the case of constrained perpendicular triplet.

\section{APPENDIX A}

\section{An analysis of the "azulene effect" in the case of a double equilibrium between the perdicular triplet and the two planar triplets}

With a double equilibrium ${ }^{3} t \rightleftarrows{ }^{3} p \rightleftarrows{ }^{3} c$, the effect of azulene on the photostationary ratio of a compound such as $\beta$-MS can be evaluated quantitatively from the Scheme C 


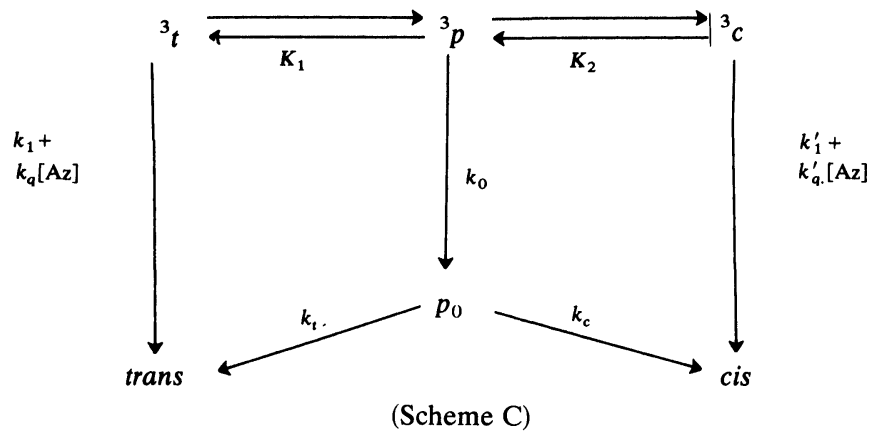

The photostationary ratio is given by:

$$
\left(\frac{\text { trans }}{\text { cis }}\right)_{s}=A \cdot \frac{\left(k_{1}+k_{q}[\mathrm{Az}]\right)\left[{ }^{3} t\right]+k_{0}\left[{ }^{3} p\right] k_{t} /\left(k_{t}+k_{c}\right)}{\left(k_{1}^{\prime}+k_{q}^{\prime}[\mathrm{Az}]\right)\left[{ }^{3} c\right]+k_{0}\left[{ }^{3} p\right] k_{c} /\left(k_{t}+k_{c}\right)}
$$

where $A$ is the ratio of the rates of energy transfer from the triplet sensitizer to the cis and trans isomers. With $\alpha, \beta$ and $\gamma$ being the fractions of triplet in ${ }^{3} t,{ }^{3} p$ and ${ }^{3} c$ conformations respectively and assuming that $k_{t}=k_{c}$ it comes:

$$
\left(\frac{\text { trans }}{\text { cis }}\right)_{s} \simeq A \cdot \frac{\alpha\left(k_{1}+k_{q}[\mathrm{Az}]\right)+0.5 k_{0} \beta}{\gamma\left(k_{1}^{\prime}+k_{q}^{\prime}[\mathrm{Az}]\right)+0.5 k_{0} \beta}
$$

With the large concentrations of azulene used in these experiments, $k_{q}[\mathrm{Az}] \gg k_{1}$ and $k_{q}^{\prime}[\mathrm{Az}] \gg k_{1}^{\prime}$ and $k_{0} \beta \approx 1 / \tau_{0}\left(\tau_{0}\right.$ being the triplet lifetime in absence of azulene). Then assuming that $k_{q}^{\prime}=k_{q}$ and with $Q=2 \tau_{0} k_{q}[\mathrm{Az}]$ :

$$
\left(\frac{\text { trans }}{\text { cis }}\right)=A(1+Q \alpha) /(1+Q \gamma)
$$

Assuming that the ${ }^{3} t,{ }^{3} p$ and ${ }^{3} c$ species are in very fast equilibrium and that therefore their population is only determined by thermodynamics, i.e., assuming $\left[{ }^{3} t\right] /\left[{ }^{3} c\right]=\exp (-\Delta H / R T)$ with $\Delta H$ being the energy difference between ${ }^{3} c$ and ${ }^{3} t$, one finally gets:

$$
\left(\frac{\text { cis }}{\text { trans }}\right)_{s} \cdot A=\frac{1+Q(1-\beta)+\exp (-\Delta H / R T)}{1+[Q(1-\beta)+1] \exp (-\Delta H / R T)}
$$

A plot of the value of the photostationary ratio vs. $(1-\beta)$, calculated with $Q=2.7\left(\tau_{0}=27 \mathrm{~ns}, k_{q}=5 \times 10^{9},[\mathrm{Az}]=0.02 \mathrm{M}\right)$ for several values of $\Delta H$ is given in Figure 5. It appears that a significant 


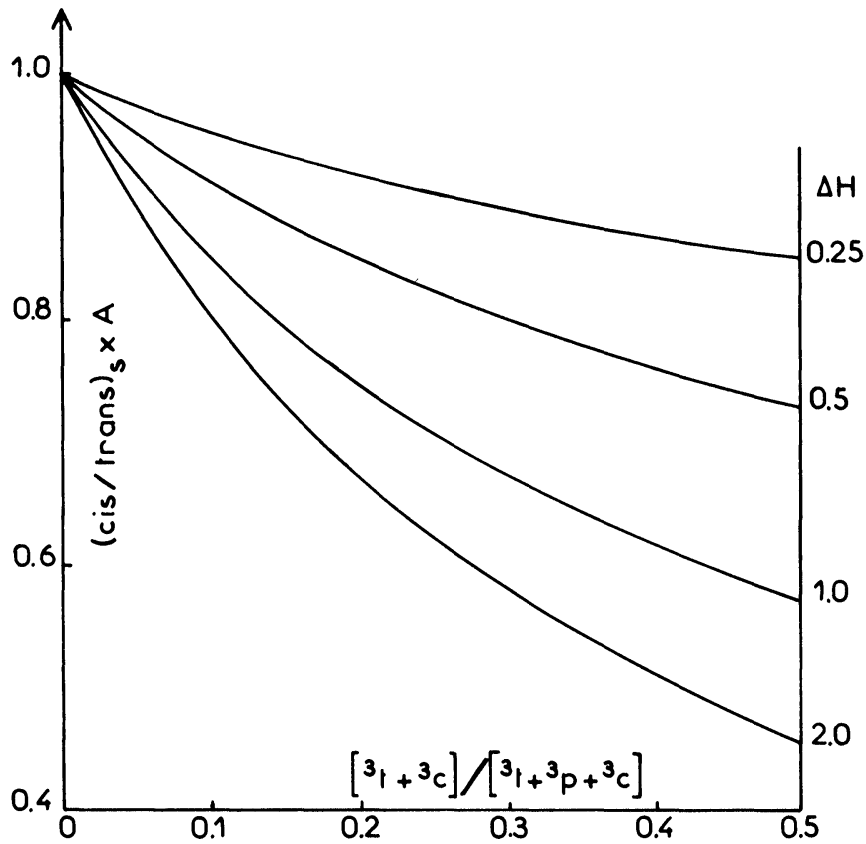

FIGURE 5 Calculated variations of photostationary ratio between the cis and trans isomers of $\beta$-methylstyrene produced by the addition of azulene $(0.02 \mathrm{M})$ as a function of fraction of planar triplets $\left({ }^{3} t\right.$ and $\left.{ }^{3} c\right)$ for several values of $\Delta H$, the difference between the energies of ${ }^{3} c$ and ${ }^{3} t$.

modification $(\geqslant 10 \%)$ of the photostationary state should be obtained if $\beta \leqslant 0.94$ as long as $\Delta H \geqslant 1 \mathrm{kcal} / \mathrm{mol}$, but with $\Delta H=0.25 \mathrm{kcal} / \mathrm{mol}$, the presence of $20-25 \%$ of planar triplet could hardly be detected by the azulene effect.

\section{APPENDIX B}

The effect of oxygen on $\phi_{c t}$ in the case of an equilibrium between the planar cis and perpendicular triplets

If a fast equilibrium, ${ }^{3} c \rightleftarrows{ }^{3} p$, does exist as shown is scheme $D$ the effect of oxygen on the yield of cis-trans sensitized photoisomerisation, $\phi_{c t}$, can be quantitatively analyzed as follows. 


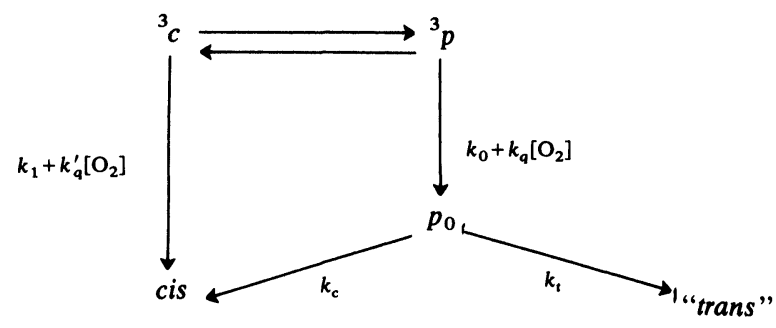

(Scheme D)

With $\gamma$ and $\beta=(1-\gamma)$ being respectively the fraction of ${ }^{3} c$ and ${ }^{3} p$ and neglecting $k_{1}$ which is much smaller than all the other rates, $\phi_{c t}$ is given by:

$$
\begin{aligned}
\phi_{0} & =k_{t} /\left(k_{t}+k_{c}\right) \quad \text { in the absence of oxygen } \\
\phi & =\phi_{0}\left[1+\gamma k_{q}^{\prime}\left[\mathrm{O}_{2}\right] / \beta\left(k_{0}+k_{q}\left[\mathrm{O}_{2}\right]\right)\right] \text { in the presence of oxygen. }
\end{aligned}
$$

This can be written as:

$$
\phi /\left(\phi_{0}-\phi\right)=\left(\beta k_{q} / \gamma k_{q}^{\prime}\right)+\left(\beta k_{0} / \gamma k_{q}^{\prime}\left[\mathrm{O}_{2}\right]\right)
$$

Then, with $\beta k_{0}=1 / \tau_{0}$ and assuming that $k_{q}=3 k_{q}^{\prime}$ as expected from spin statistical factors for the quenching by oxygen, one gets:

$$
\left.\phi /\left(\phi_{0}-\phi\right)=3 \beta / \gamma+\beta / \gamma \tau_{0} k_{q}^{\prime}\left[\mathrm{O}_{2}\right]\right)
$$

Plots of $\phi /\left(\phi_{0}-\phi\right)$ vs. $1 /\left[\mathrm{O}_{2}\right]$, drawn from the values of $\phi / \phi_{0}$ given in Table III with $k_{q}^{\prime}=3 \times 10^{9} \mathrm{M}^{-1} \mathrm{~s}^{-1}$ and $\tau_{0}=65 \mathrm{~ns}$ for PC6 or $28 \mathrm{~ns}$ for $\mathrm{PC7}$, yield the following results:

from the slopes:

$\gamma=0.67, \beta=0.33$ for PC6 and $\gamma=0.68, \beta=0.32$ for PC7

from the intercepts:

$$
\gamma=0.91, \beta=0.09 \text { for PC6 and } \gamma=0.66, \beta=0.34 \text { for PC7 }
$$

The two sets of values do not fit together in the case of PC6 and the high percentages of planar triplet predicted by this analysis for PC6 and PC7 are incompatible with other experimental results, e.g., the inefficient quenching of the PC6 triplet state by ferrocene. Therefore the decrease of $\phi_{c t}$ when the oxygen concentration increases cannot be explained by the Scheme D. 


\section{References}

1. R. Bonneau and P. Fornier de Violet, C.R. Acad. Sc. Paris 284, 631 serie C (1977).

2. R. Bonneau, J.A.C.S. 102, 3816 (1980).

3. H. Görner and D. Schulte-Frohlinde, J. Phys. Chem. 85, 1835 (1981).

4. R. Bonneau, J. Photochem. 10, 439 (1979).

5. R. A. Caldwell and C. V. Cao, J.A.C.S. 103, 3594 (1981) and 104, 6174 (1982).

6. J. Saltiel and B. Thomas, Chem. Phys. Lett. 37, 147 (1976).

7. H. E. Zimmerman, K. S. Kamm and D. P. Werthemann, J.A.C.S. 97, 3718 (1975), But recent results (R. Bonneau, J.A.C.S. 104, 2921 (1982)) indicate that this assumption is wrong in many cases, especially for styrene where $\phi_{\text {isc }} \simeq 40 \%$.

8. R. Bonneau, J. Joussot-Dubien, L. Salem and A. J. Yarwood, J.A.C.S. 98, 4329 (1976).

9. R. Bonneau, J. Joussot-Dubien, A. J. Yarwood and J. Pereyre, Tetrahedron Lett. 245 (1977).

10. R. Bensasson and E. J. Land, Trans. Faraday Soc. 67, 1904 (1971).

11. From: Solubilities of Inorganic and Organic Compounds, Vol. 1, eds. H. Stephen and T. Stephen (Pergamon Press, 1963). The solubility of NO in cyclohexane under one atmosphere pressure would be $1.8 \times 10^{-2} \mathrm{M}$. We used a lower value, $1.3 \times 10^{-2} \mathrm{M}$, because according to the same reference the solubility of NO in several other organic solvents is only slightly larger than that of $\mathrm{O}_{2}$.

12. P. J. Kropp, E. J. Reardon Jr., Z. L. F. Gaibel, K. F. Williard and J. H. Hattaway, J.A.C.S. 95, 7058 (1973).

13. R. E. Schwerzel and R. A. Caldwell, J.A.C.S. 95, 1382 (1973).

14. S. Lazare, R. Bonneau and R. Lapouyade, J. Phys. Chem. in press.

15. A. J. G. Barwise, A. A. Gorman and M. A. J. Rodgers. Chem. Phys. Lett. 38, 313 (1976).

16. R. A. Caldwell and R. Pac. Chem. Phys. Lett. 64, 303 (1979).

17. R. D. Small Jr. and J. C. Scaiano, J. Phys. Chem. 81, 2126 (1977).

18. B. Herran, Thesis, University of Bordeaux, (1980).

19. O. L. J. Gijzeman, F. Kaufman and G. Porter, Trans. Faraday Soc. II 69, 727 (1973). Reported $k_{q}=0.8 \times 10^{7} \mathrm{M}^{-1} \mathrm{~s}^{-1}$ for anthracene in hexane but they assumed $|\mathrm{NO}|=3.4 \times 10^{-2} \mathrm{M}$ under a 1 atmosphere pressure. Values of $k_{q}|\mathrm{NO}|$ from their results and from this work are in good agreement.

20. Handbook of Photochemistry, ed. S. M. Murov (Marcel Dekker, 1973).

21. T. Arai, T. Karatsu, H. Sakuragi and K. Tokumaru, Chemistry Lett. 1377 (1981). 